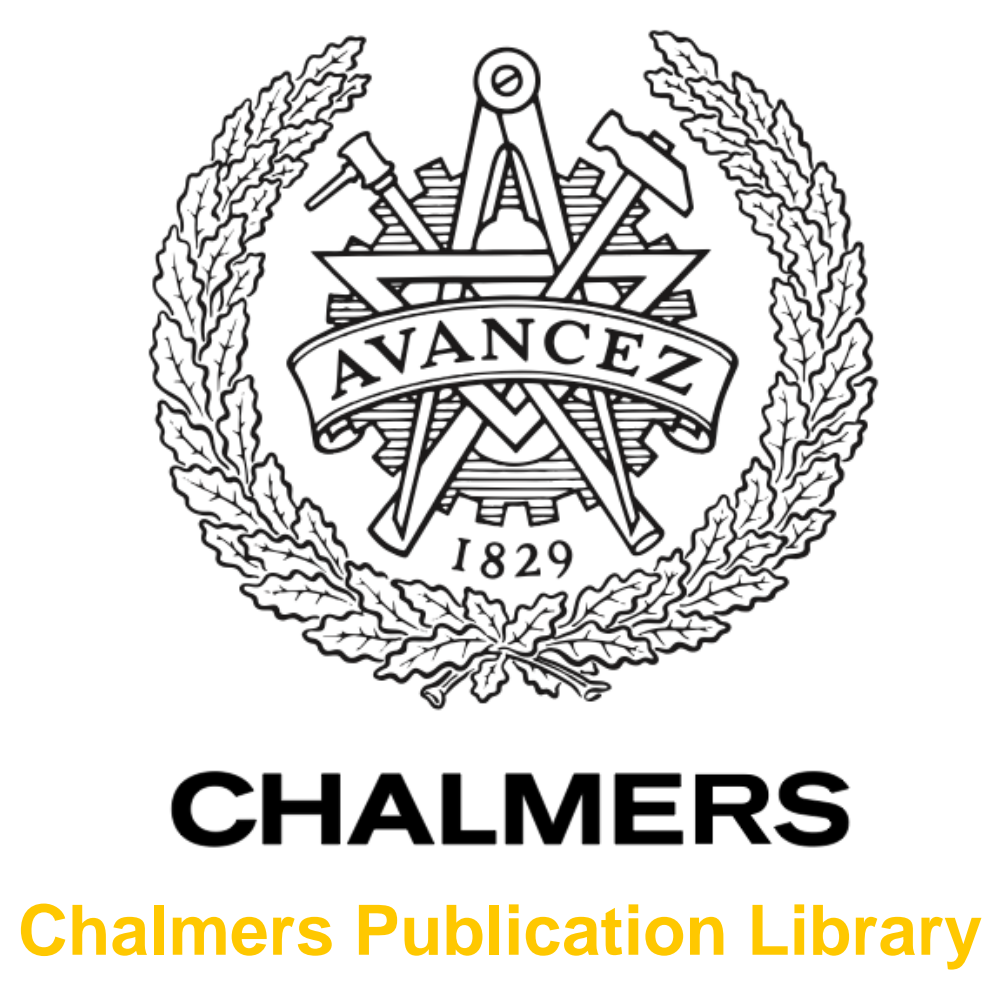

\title{
Sound Field Coherence Behind a Low Barrier in a Turbulent Atmosphere without the Presence of a Ground Surface
}

This document has been downloaded from Chalmers Publication Library (CPL). It is the author's version of a work that was accepted for publication in:

Acta Acustica United with Acustica (ISSN: 1610-1928)

Citation for the published paper:

Forssén, J. (2010) "Sound Field Coherence Behind a Low Barrier in a Turbulent Atmosphere without the Presence of a Ground Surface". Acta Acustica United with Acustica, vol. 96(5), pp. 814-822.

http://dx.doi.org/10.3813/AAA.918339

Downloaded from: http://publications.lib.chalmers.se/publication/127901

Notice: Changes introduced as a result of publishing processes such as copy-editing and formatting may not be reflected in this document. For a definitive version of this work, please refer to the published source. Please note that access to the published version might require a subscription. 


\title{
Sound field coherence behind a low barrier in a turbulent atmosphere without the presence of a ground surface
}

Jens Forssén Chalmers University of Technology, Göteborg, Sweden jens.forssen@chalmers.se

\begin{abstract}
The turbulence in the atmospheric boundary layer causes the sound propagation to take place in a random medium. The influence on the sound can be strong especially in acoustic shadow regions, which can be caused by refraction, ground attenuation or geometry, e.g. by a noise barrier. Here, situations with a noise barrier that is low in comparison with its distance to source and receiver are studied in two dimensions. Previous studies on turbulence effects have been made on the increased sound level in shadow regions as well as on the reduced coherence in line-of-sight situations and in shadow regions caused by refraction. Here, the main focus is instead on the reduced coherence of the sound field in the shadow region behind a noise barrier. Analytical as well as numerical results are presented whose implications are relevant for future studies on the sound level increase in shadow regions when the receiver is located above a ground surface. As one of the results it is concluded that the turbulence causes a larger coherence loss for screened cases than for line-of-sight cases.
\end{abstract}

\section{Introduction}

The atmospheric turbulence affects the outdoor sound. Especially in sound shielding situations the turbulence may gain large influence. For flat geometries the effects of multiple scattering grows stronger. (A geometry is here seen as flat when the barrier, or another shielding object, is low in comparison to its distance to the source and to the receiver, so that the diffraction angle is small. Otherwise the geometry is seen as steep.) The modelling here is directed toward flat geometries and could be seen as a complement to models based on single-scattering approximations, which are better applicable to steep geometries, like scattering cross-section models. 
In a previous paper analytical work was carried out on the sound level increase behind a barrier in a turbulent atmosphere [1]. In the present paper a similar approach is used for studying the coherence of the sound field behind the barrier. In both papers it is assumed that there is no influence of a ground surface, or that the source and receiver are placed on an acoustically hard ground. The coherence of the sound field is of interest when predicting the sound level behind a barrier for situations with multiple sound paths, since it determines the interference pattern and hence affects the sound pressure level. The results may be of interest to a variety of screening applications, e.g. predicting environmental noise levels, detecting objects, measuring sound radiation from objects, remote sensing of the atmosphere and beamforming applications in shielded situations. In general, for sound propagation over a barrier, such situations may occur for the cases of multiple sources radiating to a single receiver, a single source radiating to multiple receivers, i.e. a microphone array, and a single source radiating to a single receiver where one or both are located above a ground surface. The first two situations, which are equivalent by reciprocity, are studied in the present paper in the absence of a ground surface whereas the third situation belongs to future work, which hopefully could take its starting point in the work presented here. In the present state, the approach shown here gives insight into the general behaviour of sound field coherence behind a shielding object in a random medium. In addition, the results may be of use for further development of prediction models applicable to shadow zones created by refraction as well as by diffraction.

Significant work has previously been devoted to the study of the increase in sound level due to turbulence for refractive shadows (e.g. [2, 3]) and for screened areas (e.g. $[4,5,6])$. For line-of-sight propagation through a random medium above ground, the change in sound level and the reduced coherence has been extensively studied (e.g. [7, 8, 9]). The coherence of sound field in shadow regions has attracted less interest. However, works by Havelock et al. $[10,11]$ have been carried out for situations involving upward refraction, mainly focussing on beamforming, and as one result it is concluded that the coherence is reduced compared to line-of-sight propagation. The effect of sound field coherence on the mean sound level at a receiver located above a ground surface in a shadow region created by a barrier has previously been touched upon in analytical approximations (e.g. [12]) and indirectly treated in numerical work (e.g. [5]).

The results presented here uses a previously developed substitute sources method as starting point [13]. The initial formulation of the method (equation 5), here referred to as 
the full method, involves a double integral and the numerical solution is computationally demanding; it could be used as a reference method but is too heavy for a fast engineering prediction tool, e.g. for traffic noise mapping. The final result presented here is an analytical solution (equation 19). As an intermediate step an expression (equation 17), containing a single integral, is found, whose numerical implementation could be used as an engineering prediction tool, here referred to as the fast method. In comparison to the fast method, the analytical solution involves further approximations and has a smaller range of validity, but is helpful for the understanding and might be useful in some applications. Concerning the applicability to microphone arrays with phase shifted channels, the results down to and including the fast method (equation 17), are relevant whereas the analytical solution (equation 19) is not applicable. In a previous work [1], the same starting point was used as here, and the turbulence-caused sound pressure level increase behind a barrier was studied. A similar approach is taken here, with the main difference that the result presented here is more general; it reproduces the result for a single receiver in the limit of collocated receivers.

It could be noted that the full method can be applied to any barrier height [14], whereas here, for reduced computation time, the Kirchhoff approximation is used, which makes it applicable to only flat geometries. For the cases without turbulence, the Kirchhoff approximation is found to give an error smaller than $1 \mathrm{~dB}$ for diffraction angles of about $12^{\circ}$ or smaller [13]. For a barrier in a flat geometry, the surface properties of the barrier are of comparably smaller importance than for a barrier in a steep geometry [15]. Therefore the solution for a thin screen presented here can be seen as an approximation for an arbitrary barrier, concerning shape and surface material, in a flat geometry.

It should however be pointed out that for a barrier that is placed on a ground surface, higher order diffraction terms will come into play if the barrier is low compared with the sound wavelength. This effect is not taken account of in the present paper, where no ground surface is modelled. The effect would give a lower limit to the barrier height, whereas the Kirchhoff approximation gives an upper limit. However, since the turbulence effects generally are of importance at higher frequencies, the results presented here are expected to have a useful range of validity in their further development to include a ground surface.

In the present paper only two-dimensional (2-D) propagation modelling is made. Previous studies indicate that 2 -D modelling is sufficient for a variety of situations where the increased sound level behind a barrier due to turbulence is investigated [14]. The next Section describes the theory in brief; for further details the reader is referred to previous work 
[1]. In Section 3 numerical examples are shown and in Section 4 conclusions are drawn. In the Appendix the used turbulence models are described.

\section{Theory}

When the sound propagation through a random medium takes place along two or more different paths, the total sound pressure depends on the coherence of the sound pressures of the different paths. The multiple paths can be due to a ground surface that reflects the sound wave from the source, creating a direct and a ground reflected wave, or there could be multiple receiver positions from which the sound pressures are added, as relevant for microphone arrays (or, reciprocally, for multiple sources). Here, however, only cases without a ground surface are considered, i.e. multiple sources radiating to a single receiver or a single source radiating to multiple receivers. If the sound pressure from two paths, $p=p_{1}+p_{2}$, is taken as an example (see Figure 1), the long term energy average, which is of interest here, can be written as

$$
\left\langle|p|^{2}\right\rangle=\left\langle\left(p_{1}+p_{2}\right)\left(p_{1}+p_{2}\right)^{*}\right\rangle=\left\langle\left|p_{1}\right|^{2}\right\rangle+\left\langle\left|p_{2}\right|^{2}\right\rangle+2 \Re\left\{\left\langle p_{1} p_{2}^{*}\right\rangle\right\}
$$

where ${ }^{*}$ means the complex conjugate and $\Re\{\}$ means the real part. The total squared pressure average can thus be written as three terms: two energy terms (positive) and one cross term (positive or negative). If the cross term is non-zero, interference patterns in $\left\langle|p|^{2}\right\rangle$ can be created, whereas in the limit of strong turbulence effect, the cross term tends to zero and interference patterns disappear. The cross-term may be described by a (complex-valued) coherence function

$$
C=\frac{\left\langle p_{1} p_{2}^{*}\right\rangle}{\sqrt{\left\langle\left|p_{1}\right|^{2}\right\rangle\left\langle\left|p_{2}\right|^{2}\right\rangle}}
$$

For line-of-sight situations it is common to assume that the energy terms are the same as for the corresponding non-turbulent condition, which can be motivated by an energy conservation argument and assuming homogeneous and isotropic turbulence as well as neglectable effects of air attenuation. However, for a shielded receiver position, a random medium can cause an increased acoustic energy on average, and the same energy conservation argument cannot be used. Hence, it is relevant to study the effect of a random medium on the energy terms as well as on the cross term of equation (1). An analytical formulation of the cross term may however serve as the general case since the energy terms can be found from the special case of collocated paths of $p_{1}$ and $p_{2}$ (i.e. with $\rho=0$ in Figure 1). 
Using the Kirchhoff approximation (valid for small diffraction angles) and a far-field approximation, the sound pressure behind a barrier, $p_{\mathrm{H}}$, in an otherwise free field can be written by using the Rayleigh integral in two dimensions, relative to free field, $p_{\text {free, }}$ as:

$$
\frac{p_{\mathrm{H}}}{p_{\text {free }}}=\sqrt{\frac{k\left(d_{S}+d_{R}\right)}{2 \pi}} d_{S} \int_{\mathrm{H}}^{\infty} \frac{\mathrm{e}^{-\mathrm{j} k\left(R_{S}+R_{R}\right)}}{R_{S}^{3 / 2} R_{R}^{1 / 2}} \mathrm{~d} y .
$$

See Figure 2 for geometrical definitions and Ref. [1] for details on how to derive equation (3).

For two receivers equation (3) can be used together with a (real-valued) mutual coherence function (MCF), $\Gamma$, to formulate a cross-term coefficient for the case of a turbulent atmosphere. The MCF can be defined as

$$
\Gamma_{12}=\frac{\left\langle p_{1} p_{2}^{*}\right\rangle+\left\langle p_{1}^{*} p_{2}\right\rangle}{\left\langle\hat{p}_{1} \hat{p}_{2}^{*}\right\rangle+\left\langle\hat{p}_{1}^{*} \hat{p}_{2}\right\rangle}=\frac{\Re\left\{\left\langle p_{1} p_{2}^{*}\right\rangle\right\}}{\Re\left\{\left\langle\hat{p}_{1} \hat{p}_{2}^{*}\right\rangle\right\}}
$$

where $p_{1}$ and $p_{2}$ are the fluctuating pressure amplitudes in the turbulent atmosphere and $\hat{p}_{1}$ and $\hat{p}_{2}$ are the amplitudes in a free field without turbulence. The cross-term coefficient can then be written as

$$
C_{\mathrm{H}, \mathrm{rel}}=\frac{\left\langle p_{H 1} p_{H 2}^{*}\right\rangle}{\left|p_{\text {free }}\right|^{2}}=A \int_{\mathrm{H}}^{\infty} \int_{\mathrm{H}}^{\infty} \Gamma\left(y_{1}, y_{2}\right) D\left(y_{1}, y_{2}\right) \mathrm{d} y_{1} \mathrm{~d} y_{2}
$$

where $D\left(y_{1}, y_{2}\right)=\frac{\mathrm{e}^{-\mathrm{j} k\left(R_{S 1}+R_{R 1}-R_{S 2}-R_{R 2}\right)}}{R_{S 1}^{3 / 2} R_{S 2}^{3 / 2} R_{R 1}^{1 / 2} R_{R 2}^{1 / 2}}, A=\frac{k\left(d_{S}+d_{R}\right) d_{S}^{2}}{2 \pi}, R_{S 1}=\sqrt{d_{S}^{2}+y_{1}^{2}}, R_{S 2}=\sqrt{d_{S}^{2}+y_{2}^{2}}$, $R_{R 1}=\sqrt{d_{R}^{2}+\left(y_{1}-\rho / 2\right)^{2}}, R_{R 2}=\sqrt{d_{R}^{2}+\left(y_{2}+\rho / 2\right)^{2}}$ and where $p_{\text {free }}$ is taken at $y=0$, i.e. at the horizontal line through the source and mid height between the two receivers. (See Figure 2. For other cases, a rotation transformation can be used to reach the case with $y=0$ at mid height between the receivers, applicable to flat geometries.) Here, a known MCF can be used since we have line-of-sight propagation from the barrier to the receiver. (Adaptation of an analytically known MCF to general cases with two source positions and/or two receiver positions are described in Ref. [16] for flat geometries.) It should be noted that the atmosphere is turbulent only for $d>d_{S}$, where $d$ is the horizontal range measured from the source, which means that there is turbulence only after the barrier.

The above equation (5) is solved numerically, referred to as the full method, whereby the integrations can be seen as a double sum of contributions from discrete sources. That is to say, it is here assumed that the total coherence (between $p_{1}$ and $p_{2}$ ) can in general be written as a sum of coherences between partial sources, an assumption here referred to as the substitute-sources assumption.

For the further analysis, a rewritten form of the MCF is used [16], as

$$
\Gamma=\mathrm{e}^{-2 \gamma x}+\left(1-\mathrm{e}^{-2 \gamma x}\right) \hat{\Gamma}
$$


where $\gamma$ is the extinction coefficient for the sound field in the turbulent atmosphere (see equation 24 in Appendix) and whereby $\hat{\Gamma}$ becomes a function decaying toward zero for increasing $\rho$ (since $\hat{\Gamma}=\left(\Gamma-\mathrm{e}^{-2 \gamma x}\right) /\left(1-\mathrm{e}^{-2 \gamma x}\right)$ and $\Gamma$ tends to $\left.\mathrm{e}^{-2 \gamma x}\right)$. For turbulence only behind the barrier, the range $x$ of propagation through turbulence should strictly be set to $d_{R}$, as is done here. However, $x$ could in engineering applications be approximated as the total distance, $x=d_{S}+d_{R}$, which is a reasonable approximation since the error can be supposed to be small when relating to the real case of interest, with turbulence all the way from the source to the receiver. Using equation (6) in (5) gives

$$
C_{\mathrm{H}, \mathrm{rel}}=\mathrm{e}^{-2 \gamma x} A \int_{H}^{\infty} \int_{H}^{\infty} D \mathrm{~d} y_{1} \mathrm{~d} y_{2}+\left(1-\mathrm{e}^{-2 \gamma x}\right) A \int_{H}^{\infty} \int_{H}^{\infty} \hat{\Gamma} D \mathrm{~d} y_{1} \mathrm{~d} y_{2} .
$$

The first term can be identified as an integration for a non-turbulent condition, denoted $C_{\mathrm{H}, \mathrm{rel}}^{0}$, i.e.

$$
C_{\mathrm{H}, \mathrm{rel}}^{0}=A \int_{H}^{\infty} \int_{H}^{\infty} D \mathrm{~d} y_{1} \mathrm{~d} y_{2}
$$

If the integration domain above (i.e. $\left.y_{1}, y_{2} \epsilon(H, \infty)\right)$ is extended to infinity (i.e. $y_{1}, y_{2} \epsilon(-\infty, \infty)$ ), the result for an unobstructed terrain should be retained according to the substitute-sources assumption described above, i.e.

$$
\left.C_{\mathrm{H}, \text { rel }}\right|_{H=-\infty}=\frac{\left\langle p_{1} p_{2}^{*}\right\rangle}{\left|p_{\text {free }}\right|^{2}}=\Gamma_{12}^{0}
$$

with $\Gamma_{12}^{0}$ the known coherence function for two receivers separated a distance $\rho$ in a free space containing turbulence. (It could be noted that, for a single receiver position, i.e. $\rho=0$ in Figure 1, we have $\Gamma=1$ and then the substitute-sources assumption can be motivated by energy conservation, see Ref. [1].) The substitute-sources assumption can be taken advantage of to change the integration domain by writing

$$
\Gamma_{12}^{0}=A \int_{-\infty}^{\infty} \int_{-\infty}^{\infty} \Gamma D \mathrm{~d} y_{1} \mathrm{~d} y_{2}
$$

and

$$
A \int_{-\infty}^{\infty} \int_{-\infty}^{\infty} \Gamma D \mathrm{~d} y_{1} \mathrm{~d} y_{2}=2 A \Re\left\{\int_{H}^{\infty} \int_{H}^{\infty} \Gamma D \mathrm{~d} y_{1} \mathrm{~d} y_{2}+\int_{-\infty}^{\infty} \int_{0}^{H+|v|} \Gamma D \mathrm{~d} u \mathrm{~d} v\right\}
$$

where the substitution $y_{1}+y_{2}=2 u, y_{1}-y_{2}=v$ is used together with the consequence of the integrand, $\Gamma D$, being Hermitian (symmetric real part and anti-symmetric imaginary part). In Figure 3 the two integration domains of the right hand side of equation (11) are denoted as $\widetilde{W}_{H}$ and $\widetilde{W}_{C}$, respectively (see also Ref. [1]). One then solves for the first term of the right 
hand side of equation (11), corresponding to $\widetilde{W}_{H}$ in Figure 3. By in this way using equation (11) in the last term of equation (7), with $\Gamma$ replaced by $\hat{\Gamma}$ and, analogously, $\Gamma_{12}^{0}$ replaced by $\hat{\Gamma}_{12}^{0}$, one can write

$$
\Re\left\{C_{\mathrm{H}, \mathrm{rel}}\right\}=\mathrm{e}^{-2 \gamma x} \Re\left\{C_{\mathrm{H}, \mathrm{rel}}^{0}\right\}+\left(1-\mathrm{e}^{-2 \gamma x}\right)\left(\frac{1}{2} \hat{\Gamma}_{12}^{0}-A \int_{-\infty}^{\infty} \int_{0}^{H+|v|} \hat{\Gamma}(u, v) \Re\{D\} \mathrm{d} u \mathrm{~d} v\right) .
$$

For a flat geometry the dependence of $\Gamma(u, v)$ on $u$ is weak and $\Gamma(u, v)$ can be approximated as a function of $v$ only, i.e. $\hat{\Gamma}(u, v) \approx \hat{\Gamma}(v)$. This is used to simplify the above expression and moving $\hat{\Gamma}(v)$ outside the integral with respect to $u$, which then is written out as

$$
\int_{0}^{H+|v|} \Re\{D\} \mathrm{d} u=\int_{0}^{H+|v|} \frac{\cos \left[k\left(R_{S 1}+R_{R 1}-R_{S 2}-R_{R 2}\right)\right]}{R_{S 1}^{3 / 2} R_{S 2}^{3 / 2} R_{R 1}^{1 / 2} R_{R 2}^{1 / 2}} \mathrm{~d} u .
$$

In order to analytically solve the integral with respect to $u$, it is further assumed that the integral in equation (13) is dominated by contributions from low positions, i.e. $\frac{\left|y_{1}\right|}{d_{S}}, \frac{\left|y_{2}\right|}{d_{S}}$, $\frac{\left|y_{1}-\rho / 2\right|}{d_{R}}, \frac{\left|y_{2}+\rho / 2\right|}{d_{R}} \ll 1$. The argument of the cos-function, i.e. the phase difference, can then be approximated using

$$
\begin{aligned}
& R_{S 1}-R_{S 2}=\sqrt{d_{S}^{2}+y_{1}^{2}}-\sqrt{d_{S}^{2}+y_{2}^{2}} \approx \frac{y_{1}^{2}-y_{2}^{2}}{2 d_{S}}=\frac{u v}{d_{S}} \\
& R_{R 1}-R_{R 2}=\sqrt{d_{R}^{2}+\left(y_{1}-\rho / 2\right)^{2}}-\sqrt{d_{R}^{2}+\left(y_{2}+\rho / 2\right)^{2}} \approx \frac{y_{1}^{2}-y_{2}^{2}-\rho\left(y_{1}+y_{2}\right)}{2 d_{R}}=\frac{u(v-\rho)}{d_{R}} .
\end{aligned}
$$

In the denominator of the integrand approximations are used according to

$$
R_{S 1}, R_{S 2} \approx d_{S}, \quad R_{R 1}, R_{R 2} \approx d_{R}
$$

Applying the above approximations $(14,15)$ to equation $(13)$ gives

$$
\begin{aligned}
& \int_{0}^{H+|v|} \frac{\cos \left[k\left(R_{S 1}+R_{R 1}-R_{S 2}-R_{R 2}\right)\right]}{R_{S 1}^{3 / 2} R_{S 2}^{3 / 2} R_{R 1}^{1 / 2} R_{R 2}^{1 / 2}} \mathrm{~d} u= \\
& =\int_{0}^{H+|v|} \frac{\cos \left[k u\left(\frac{v}{d_{S}}+\frac{v-\rho}{d_{R}}\right)\right]}{d_{S}^{3} d_{R}} \mathrm{~d} u=\frac{\sin \left[k(H+|v|)\left(\frac{v}{d_{S}}+\frac{v-\rho}{d_{R}}\right)\right]}{d_{S}^{3} d_{R} k\left(\frac{v}{d_{S}}+\frac{v-\rho}{d_{R}}\right)} .
\end{aligned}
$$

Using the above result, equation (12) can be rewritten as

$$
\begin{aligned}
& \Re\left\{C_{\mathrm{H}, \mathrm{rel}}\right\}=\mathrm{e}^{-2 \gamma x} \Re\left\{C_{\mathrm{H}, \mathrm{rel}}^{0}\right\}+ \\
& +\left(1-\mathrm{e}^{-2 \gamma x}\right)\left(\frac{1}{2} \hat{\Gamma}_{12}^{0}-\frac{d_{S}+d_{R}}{2 \pi d_{S} d_{R}} \int_{-\infty}^{\infty} \hat{\Gamma} \frac{\sin \left[k(H+|v|)\left(\frac{v}{d_{S}}+\frac{v-\rho}{d_{R}}\right)\right]}{\frac{v}{d_{S}}+\frac{v-\rho}{d_{R}}} \mathrm{~d} v\right)
\end{aligned}
$$


The above equation (17), with a single integral, may be a useful result since its numerical solution (here called the fast method) is less computationally heavy than the numerical solution of the full problem, which contains a double integral (equation 5).

In order to reach an analytical solution of the problem, the above equation is further simplified. To do this, strong turbulence effects are assumed, i.e. that $\hat{\Gamma}$ goes to zero fast enough to make the integrand in equation (17) contribute only for small arguments of the sin-function, and an approximation as $\sin (x) \approx x$ is used, assuming $|x| \ll 2 \pi$. In addition, an approximating function is used for $\hat{\Gamma}$. In the limit of small screen heights, the effect of turbulence is assumed to be dominated by the larger scales. This is due to that the larger scales scatter in directions more near forward, which can be seen from a Bragg scattering analogy (e.g. [12]). For the largest scales the von Kármán turbulence model is well approximated by the Gaussian one as it is used here, and hence the Gaussian model could be used here to find an approximation for near-zero screen heights and strong turbulence scattering.

For this approximation, $\hat{\Gamma}(v)$ in equation (17) is replaced by an approximation of equation (25) in Appendix for $v \ll l: \Gamma^{\mathrm{G}}(v)=\exp \left(-\alpha v^{2}\right)$, with $\alpha=\left(\gamma_{T}+2 \gamma_{v}\right) 2 x /\left(3 l^{2}\right)$ [17]. (For different length scales of the temperature and the velocity fluctuations, $l_{T}$ and $l_{v}$ respectively, one can write $\alpha=2 \gamma_{T} x /\left(3 l_{T}^{2}\right)+4 \gamma_{v} x /\left(3 l_{v}^{2}\right)$.) The strong-turbulence approximation is based on the assumption that $2 \gamma x \approx 1$ or larger [17]. For the transversally separated receiver positions used here, it can be shown that $\exp \left(-\alpha v^{2}\right)$ can be replaced by $\exp \left[-\alpha\left(v^{2}+\rho v+\rho^{2}\right)\right]$, where $\rho$ is the separation distance of the receivers.

Using the two approximations above, i.e. $\sin (x) \approx x$ and $\hat{\Gamma} \approx \exp \left[-\alpha\left(v^{2}+\rho v+\rho^{2}\right)\right]$, equation (17) can be rewritten as

$$
\begin{aligned}
& \Re\left\{C_{\mathrm{H}, \mathrm{rel}}\right\}=\mathrm{e}^{-2 \gamma x} \Re\left\{C_{\mathrm{H}, \mathrm{rel}}^{0}\right\}+ \\
& +\left(1-\mathrm{e}^{-2 \gamma x}\right)\left(\frac{1}{2} \hat{\Gamma}_{12}^{0}-\frac{k\left(d_{S}+d_{R}\right)}{2 \pi d_{S} d_{R}} \int_{-\infty}^{\infty} \mathrm{e}^{-\alpha\left(v^{2}+\rho v+\rho^{2}\right)}(H+|v|) \mathrm{d} v\right) .
\end{aligned}
$$

The integral in the above equation can be solved analytically to give the final expression as

$$
\begin{aligned}
& \Re\left\{C_{\mathrm{H}, \mathrm{rel}}\right\}=\mathrm{e}^{-2 \gamma x} \Re\left\{C_{\mathrm{H}, \mathrm{rel}}^{0}\right\}+\left(1-\mathrm{e}^{-2 \gamma x}\right) \\
& \cdot\left(\frac{1}{2} \hat{\Gamma}_{12}^{0}-\frac{k\left(d_{S}+d_{R}\right)}{2 \pi d_{S} d_{R}} \frac{\mathrm{e}^{-\alpha \rho^{2}}}{\alpha}\left\{1+\mathrm{e}^{\alpha \rho^{2} / 4} \sqrt{\pi \alpha}\left[H+\frac{\rho}{2} \operatorname{erf}(\sqrt{\alpha \rho / 2})\right]\right\}\right)
\end{aligned}
$$

where $\operatorname{erf}(\cdot)$ is the error function, $\operatorname{erf}(x)=\frac{2}{\sqrt{\pi}} \int_{0}^{x} \exp \left(-t^{2}\right) \mathrm{d} t$ (see e.g. [18]). To repeat, the link between equations (19) and (1) is as follows: $\Re\left\{C_{\mathrm{H}, \text { rel }}\right\}=\Re\left\{\left\langle p_{1} p_{2}^{*}\right\rangle\right\} /\left|p_{\text {free }}\right|^{2}$, where $p_{1}=p_{1 H}$ 
and $p_{2}=p_{2 H}$, i.e. for a screen height $H$, and where $p_{\text {free }}$ is evaluated at the midpoint between the two receivers. Here, it could be pointed out that for $\rho=0$ we retain the solution for the increase due to turbulence for a single receiver (as previously reported in Ref. [1]):

$$
\begin{aligned}
& C_{\mathrm{H}, \mathrm{rel}}=\mathrm{e}^{-2 \gamma x} C_{\mathrm{H}, \mathrm{rel}}^{0}+ \\
& +\left(1-\mathrm{e}^{-2 \gamma x}\right)\left[\frac{1}{2}-\frac{k\left(d_{S}+d_{R}\right)}{2 \pi d_{S} d_{R} \alpha}(1+\sqrt{\pi \alpha} H)\right] .
\end{aligned}
$$

In the above analytical solution (equation 19), the first term corresponds to the coherent field and the second term to the incoherent field. It can be seen that the incoherent part increases with the extinction coefficient, $\gamma$, as expected. This can also be seen in the formulation of the fast method. As also expected, an increased separation, $\rho$, causes a reduced coherence, which can be shown from equation (19) for small values of $\rho$. Furthermore, and of larger current interest, it can be seen that the coherence is reduced if the screen height, $H$, is increased.

Concerning the range of applicability of the analytical solution (equation 19), the inequality $|x| \ll 2 \pi$ used for $\sin (x) \approx x$ in equation (17) must hold as long as $\alpha\left(v^{2}+\rho v+\rho^{2}\right)$ in equation (18) is near 1 or smaller, where $\alpha\left(v^{2}+\rho v+\rho^{2}\right)=1$ is attained at the correlation radius. If we take $\rho=0$ as the worse case, we can identify the correlation radius, $v_{c}$, from $\alpha v_{c}^{2}=1$. Using $\rho=0, v=v_{c}$ and $|x|=\left|k\left(H+v_{c}\right)\left(1 / d_{S}+1 / d_{R}\right) v_{c}\right| \ll 2 \pi$ we can identify a limiting inequality as $\left|H+v_{c}\right| v_{c} \ll v_{\mathrm{F}}^{2}$, where $v_{\mathrm{F}}$ is the first Fresnel zone above the bar-

rier, which here is given by $v_{\mathrm{F}}^{2}=2 \pi /\left[k\left(1 / d_{S}+1 / d_{R}\right)\right]$, assuming $k d_{S}, k d_{R} \gg 1$. The limiting inequality can be separated into two inequalities, as

$$
v_{c}^{2} \ll v_{\mathrm{F}}^{2}
$$

and

$$
|H| v_{c} \ll v_{\mathrm{F}}^{2}
$$

\section{Calculated examples}

In the calculated examples, first the substitute-sources assumption is checked numerically, i.e. equation (10). For this, a MCF is used according to equation (23) in Appendix. Here, all calculated examples uses the von Kármán turbulence model. Since the first part of the propagation, $d_{S}$, is without turbulence in the calculations, the MCF according to equation (23) is corrected to model the same situation. (For such a correction, see e.g. Ref. [16]; one uses 
the multiplicative property of the MCF over range, which is a result of the approximations used when deriving the MCF, see e.g. Ref. [19]). All the calculated examples are for a total range of $1 \mathrm{~km}$, with $d_{S}=100 \mathrm{~m}$ and $d_{R}=900 \mathrm{~m}$. In Figure 4 the results from the numerical check of the substitute-sources assumption are shown as a function of the receiver separation distance, where the values of $\Gamma_{12}^{0}$ from equation (23) can be seen to be well reproduced. Here the frequency $f=1000 \mathrm{~Hz}$, a turbulence length scale of $L_{0}=10 \mathrm{~m}$ and different strengths of velocity turbulence have been used, from $C_{v}^{2}=1 \mathrm{~m}^{4 / 3} \mathrm{~s}^{-2}$, which can be seen as strong atmospheric turbulence, down to $C_{v}^{2}=0.001 \mathrm{~m}^{4 / 3} \mathrm{~s}^{-2}$ (with $C_{T}^{2}=0$ and hence $\gamma_{T}=0$ ). It should be noted that scaling laws can be used, whereby this and the following calculated examples can be seen to cover also other cases, e.g. another total range [1].

Next are shown some examples of the absolute value of the coherence, as defined in equation (2), for different screen heights, $H$ (see Figure 5). It can be seen that the coherence is reduced the higher the barrier is. Hence, the turbulence has a stronger coherence reducing effect for screened cases than for unscreened cases, similarly to previous results for upward refraction [10]. A similar result is shown in Figure 6, where the absolute value of the coherence is instead plotted as a function of frequency for a fixed receiver separation distance of $1 \mathrm{~m}$.

The main interest here is however the real part of the coherence and its modelling by the fast method and the analytical solution compared with the full method. In Figures 7 and 8 some calculated examples are plotted for the full method and the fast method showing good agreement and an increasing accuracy for lower screens height. It can be seen that the real part of the coherence can show an oscillatory pattern at higher frequencies and larger screen heights (Figure 8). This can be seen as a damped version of the oscillatory pattern of the corresponding situation without turbulence (shown by the thinner lines).

The following six Figures 9-14 display the results from a parametric study to investigate the limits of applicability of the analytical solution. The analytical solution is then plotted only for the cases where it is expected to be applicable according to the inequalities stated above, i.e. equations (21 and 22). The parametric study used the following range of parameter values: $C_{v}^{2}=10^{-3}, 10^{-2}, 10^{-1}$, and $1 \mathrm{~m}^{4 / 3} \mathrm{~s}^{-2}$ (with $C_{T}^{2}=0$ ); $L_{0}=1,10$, and $10^{2} \mathrm{~m}$; and $f=125,250,500,1000$, and $2000 \mathrm{~Hz}$. It should be noted that even though some of the chosen values correspond to unrealistically strong turbulence situations, the use of scaling properties would change the range of realistic values. For the chosen set of parameter values only six cases fulfill a chosen condition of $v_{c}^{2} / v_{\mathrm{F}}^{2}<0.1$ (from equation 21). In the plots of the 
corresponding results, the screen height has been limited to $H<0.1 v_{\mathrm{F}}^{2} / v_{c}$ (from equation 22), for the possible values of $H=-10,0,5,10$ and $20 \mathrm{~m}$. (See figure captions for values of parameters as well as of $v_{c}$ and $v_{\mathrm{F}}$.) In Figures 9-14, it can be seen that the analytical solution approximates the results from the full method with a reasonable accuracy for the cases where it is expected to be valid. In Figure 13 the limiting condition $0.1 v_{\mathrm{F}}^{2} / v_{c}$ attains its lowest value $(1.2 \mathrm{~m})$, whereby the error of the analytical solution is larger than for the other cases.

\section{Conclusions}

Modelling is made here to study the influence of atmospheric turbulence on the sound field coherence in the shadow region behind a noise barrier. The modelling is made for a situation without a ground surface and for a flat geometry, i.e. for a barrier that is low in comparison with its distance to the source and to the receiver. For flat geometries the influence of multiple scattering grows in importance, whereby the modelling made here can be seen as complementing single-scattering modelling. The analysis is based on an assumption that the total coherence can be described as a sum of coherences of partial sources. The validity of this substitute-sources assumption is supported by the numerical results.

Three different models are presented. Two of them are suitable to numerical solution and the final one is an analytical solution. The computationally less expensive of the numerical models showed good overall agreement with the more exact model and may be useful in engineering applications like mapping of community noise. The applicability of the analytical solution was shown to be in accordance with the theoretically derived limits, which is for cases of low screen heights and with strong turbulence influence, i.e. a small correlation radius compared with the size of the first Fresnel zone.

It can be concluded from both the analytical and the numerical results that the coherence drops with increasing barrier height. Hence, the turbulence has a stronger coherence reducing effect for screened cases than for unscreened cases, similarly to what has been shown previously for upward refraction. Concerning further work, the approach used here seems promising and a natural following step is the inclusion of a ground surface. 


\section{Acknowledgments}

The work behind this paper has partially been funded by the Swedish Foundation for Strategic Environmental Research (MISTRA). The author also wishes to thank Maarten Hornikx and Wolfgang Kropp for valuable discussions.

\section{Appendix}

The mutual coherence function (MCF) will in general depend on the choice of turbulence model, its parameter values, the transversal separation, $v$, the distance of propagation, $x$, and the sound frequency, $f$. Here, the starting point is the von Kármán turbulence model with MCF for spherical wave propagation as

$$
\begin{aligned}
\Gamma^{\mathrm{vK}}(v)=\exp \{- & \frac{2 x}{K_{0} v} \int_{0}^{K_{0} v} \gamma_{T}\left[1-\frac{2^{1 / 6} t^{5 / 6}}{\Gamma(5 / 6)} K_{5 / 6}(t)\right] \\
+\gamma_{v} & {\left.\left[1-\frac{2^{1 / 6} t^{5 / 6}}{\Gamma(5 / 6)}\left(K_{5 / 6}(t)-\frac{t}{2} K_{1 / 6}(t)\right)\right] \mathrm{d} t\right\} }
\end{aligned}
$$

where $\gamma_{T}$ and $\gamma_{v}$ are the extinction coefficients of the mean field due to temperature and velocity fluctuations, respectively; $K_{0}=2 \pi / L_{0}$, where $L_{0}$ is taken as the outer scale of turbulence; $\Gamma(5 / 6) \approx 1.13$ is the gamma function; and $K_{5 / 6}$ and $K_{1 / 6}$ are modified Bessel functions of the second kind. The total extinction coefficient can be written

$$
\gamma=\gamma_{T}+\gamma_{v}=\frac{3}{10} \pi^{2} A k^{2} K_{0}^{-5 / 3}\left(\frac{C_{T}^{2}}{T_{0}^{2}}+\frac{4 C_{v}^{2}}{c_{0}^{2}}\right)
$$

where $A \approx 0.0330, T_{0}$ is the mean temperature, $c_{0}$ is the mean sound speed, and $C_{T}^{2}$ and $C_{v}^{2}$ are the structure parameters describing the strengths of temperature and velocity fluctuations, respectively. For the above formulation of $\Gamma^{\mathrm{vK}}$ Ref. [19] was used.

To the von Kármán model, the Gaussian model can be connected in a way described in Ref. [19]. It is then assumed that the integral length scales are the same for the normalized longitudinal correlation functions of the velocity fluctuations, and that the same is true for the temperature fluctuations. It is also assumed that the variances of the turbulent fields are the same. The resulting MCF for the Gaussian model can then be written

$$
\Gamma^{\mathrm{G}}(v)=\exp \left[-2 \gamma_{T} x\left(1-\frac{\Phi(v / l)}{v / l}\right)-2 \gamma_{v} x\left(1-\frac{1}{2} \frac{\Phi(v / l)}{v / l}-\frac{1}{2} \mathrm{e}^{-v^{2} / l^{2}}\right)\right]
$$

where $\Phi(v / l)=\int_{0}^{v / l} \mathrm{e}^{-s^{2}} \mathrm{~d} s$ and $l=\frac{2 \Gamma(5 / 6)}{\Gamma(1 / 3)} K_{0}^{-1} \approx 0.843 K_{0}^{-1}$. By connecting the Gaussian and the von Kármán spectra in this way, the Gaussian spectrum can be seen as modelling the 
larger scales of the turbulence. It should be noted that, for other values of the strength and the correlation length, $l$, the Gaussian spectrum can model a different range of scales. 


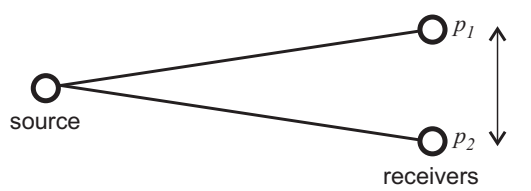

Figure 1: Geometry with two paths originating from the same source and reaching two receivers separated by a distance $\rho$.

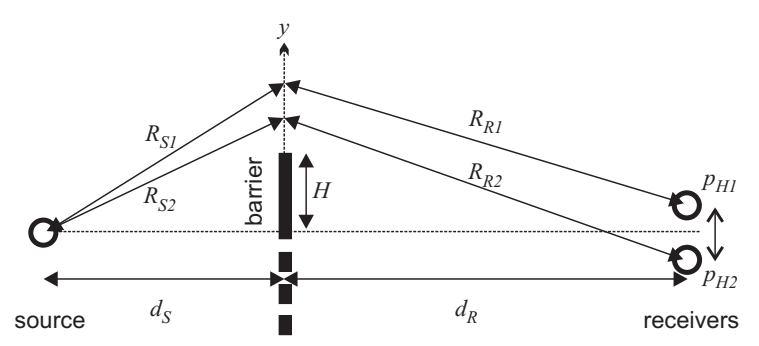

Figure 2: Definition of the barrier geometry.

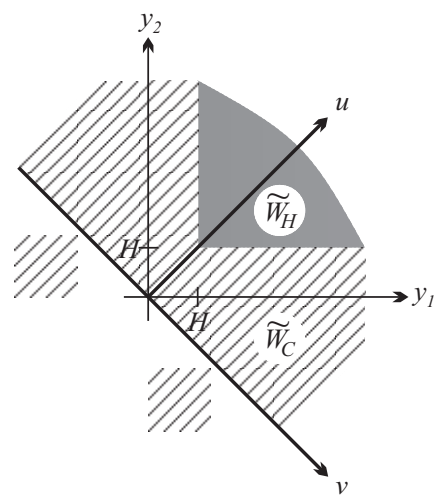

Figure 3: Integration domains used in equation (11). 


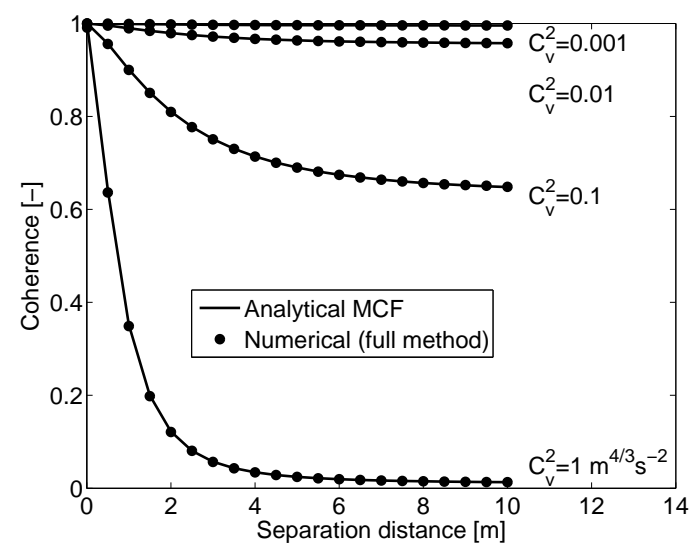

Figure 4: Numerical check that the analytically known MCF can be reproduced for the line-ofsight case. Calculated examples for $f=1000 \mathrm{~Hz}$ and for the von Kármán turbulence model with $L_{0}=10 \mathrm{~m}$ and varying strengths of velocity turbulence (see plot).

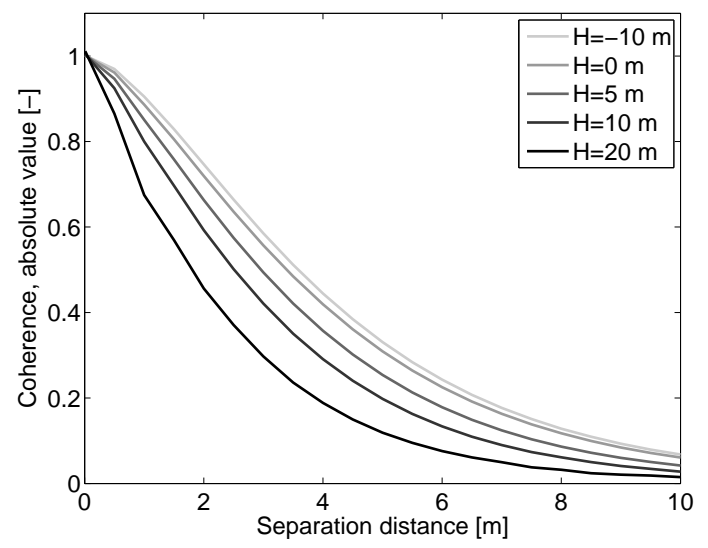

Figure 5: Absolute value of coherence for different screen heights, $H$. Calculated examples for $f=250 \mathrm{~Hz}, L_{0}=100 \mathrm{~m}$ and $C_{v}^{2}=1 \mathrm{~m}^{4 / 3} \mathrm{~s}^{-2}$. 


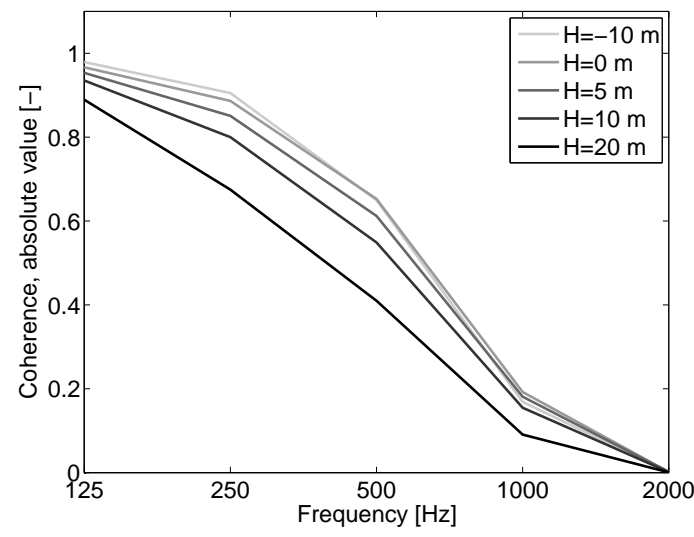

Figure 6: Absolute value of coherence for different screen heights, $H$. Calculated examples for $\rho=1 \mathrm{~m}, L_{0}=100 \mathrm{~m}$ and $C_{v}^{2}=1 \mathrm{~m}^{4 / 3} \mathrm{~s}^{-2}$.

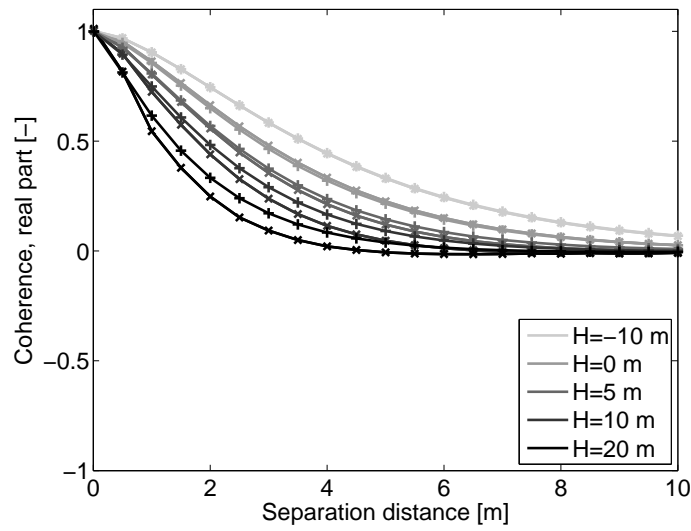

Figure 7: Real part of coherence using the full method (times signs) and the fast method (plus signs) for different screen heights, $H$. Calculated examples for $f=250 \mathrm{~Hz}, L_{0}=100 \mathrm{~m}$ and $C_{v}^{2}=1 \mathrm{~m}^{4 / 3} \mathrm{~s}^{-2}$. 


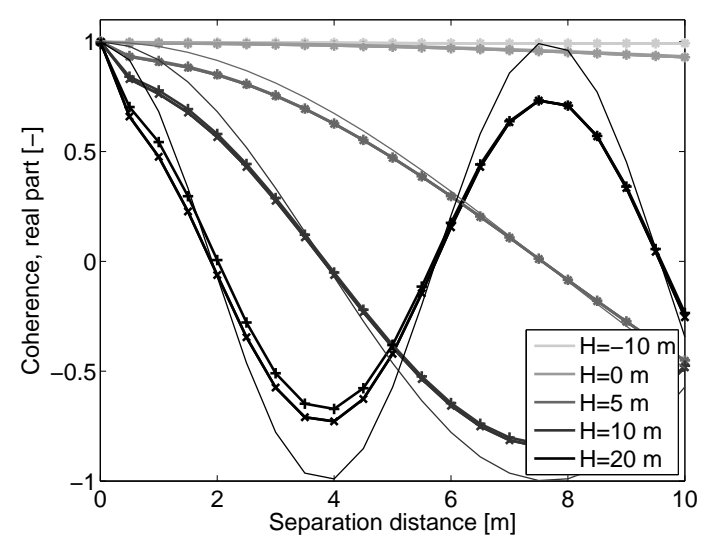

Figure 8: Real part of coherence using the full method (times signs) and the fast method (plus signs) for different screen heights, $H$. Calculated examples for $f=1000 \mathrm{~Hz}, L_{0}=1 \mathrm{~m}$ and $C_{v}^{2}=0.01 \mathrm{~m}^{4 / 3} \mathrm{~s}^{-2}$. (Results without turbulence shown by thin lines.)

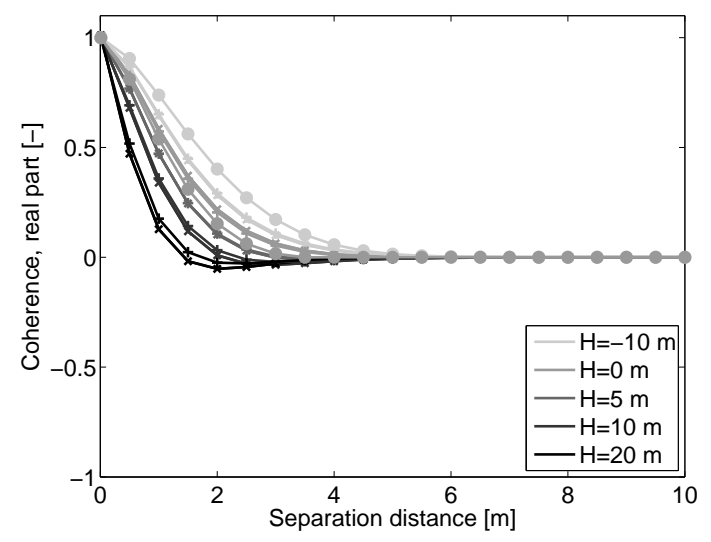

Figure 9: Real part of coherence using the full method (times signs) and the fast method (plus signs) for different screen heights, $H$. The analytical solution is plotted for the two smallest screen heights, $H=-10$ and $H=0 \mathrm{~m}$ (filled circles). $f=500 \mathrm{~Hz}, L_{0}=100 \mathrm{~m}$ and $C_{v}^{2}=1 \mathrm{~m}^{4 / 3} \mathrm{~s}^{-2}$, $v_{c}=1.7 \mathrm{~m}$ and $v_{\mathrm{F}}=7.8 \mathrm{~m}$. 


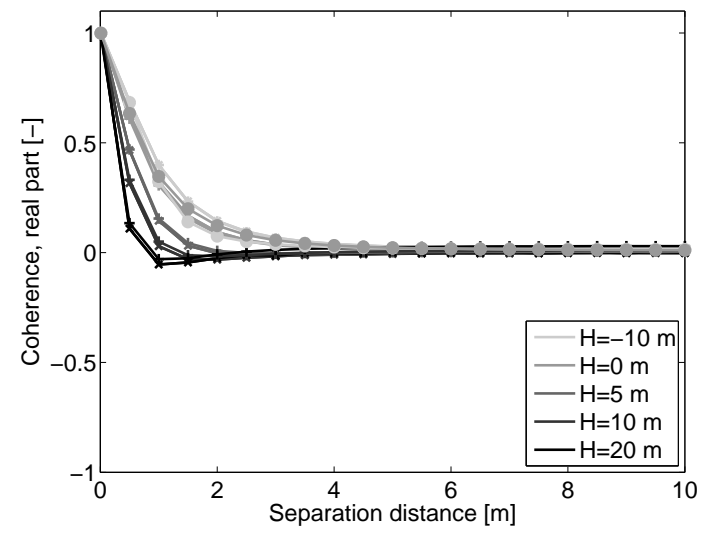

Figure 10: Real part of coherence using the full method (times signs) and the fast method (plus signs) for different screen heights, $H$. The analytical solution is plotted for the two smallest screen heights, $H=-10$ and $H=0 \mathrm{~m}$ (filled circles). $f=1000 \mathrm{~Hz}, L_{0}=10 \mathrm{~m}$ and $C_{v}^{2}=1 \mathrm{~m}^{4 / 3} \mathrm{~s}^{-2}$, $v_{c}=1.1 \mathrm{~m}$ and $v_{\mathrm{F}}=5.5 \mathrm{~m}$.

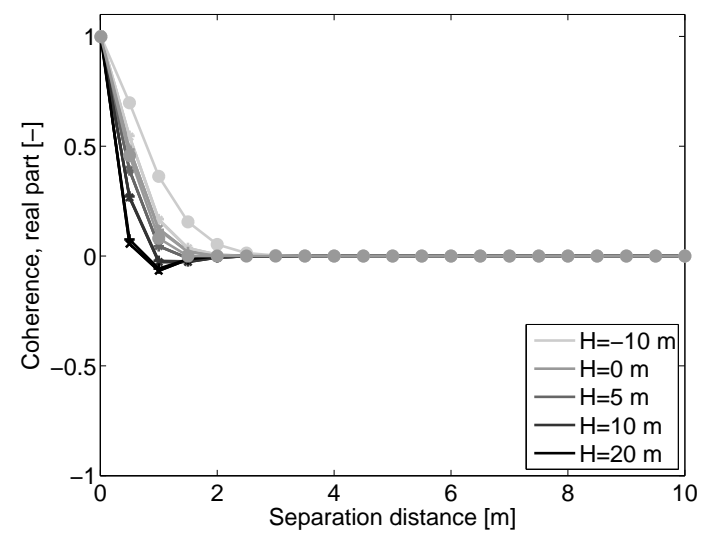

Figure 11: Real part of coherence using the full method (times signs) and the fast method (plus signs) for different screen heights, $H$. The analytical solution is plotted for the two smallest screen heights, $H=-10$ and $H=0 \mathrm{~m}$ (filled circles). $f=1000 \mathrm{~Hz}, L_{0}=100 \mathrm{~m}$ and $C_{v}^{2}=$ $1 \mathrm{~m}^{4 / 3} \mathrm{~s}^{-2}, v_{c}=0.7 \mathrm{~m}$ and $v_{\mathrm{F}}=5.5 \mathrm{~m}$. 


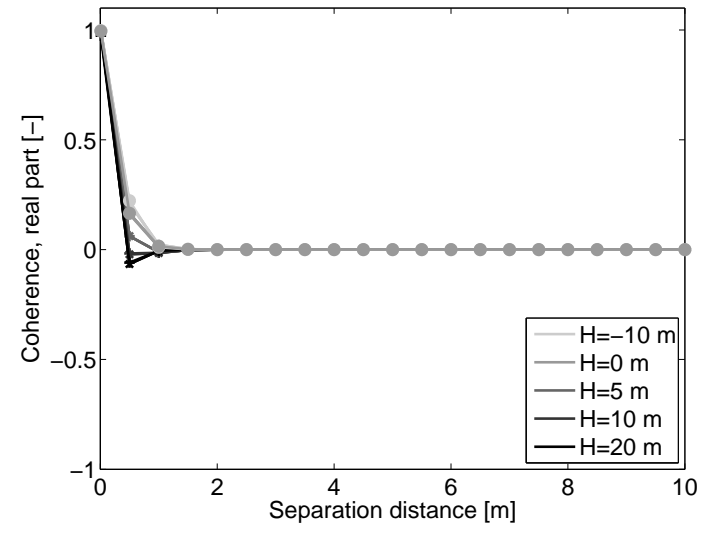

Figure 12: Real part of coherence using the full method (times signs) and the fast method (plus signs) for different screen heights, $H$. The analytical solution is plotted for the two smallest screen heights, $H=-10$ and $H=0 \mathrm{~m}$ (filled circles). $f=2000 \mathrm{~Hz}, L_{0}=10 \mathrm{~m}$ and $C_{v}^{2}=1 \mathrm{~m}^{4 / 3} \mathrm{~s}^{-2}$, $v_{c}=0.4 \mathrm{~m}$ and $v_{\mathrm{F}}=3.9 \mathrm{~m}$.

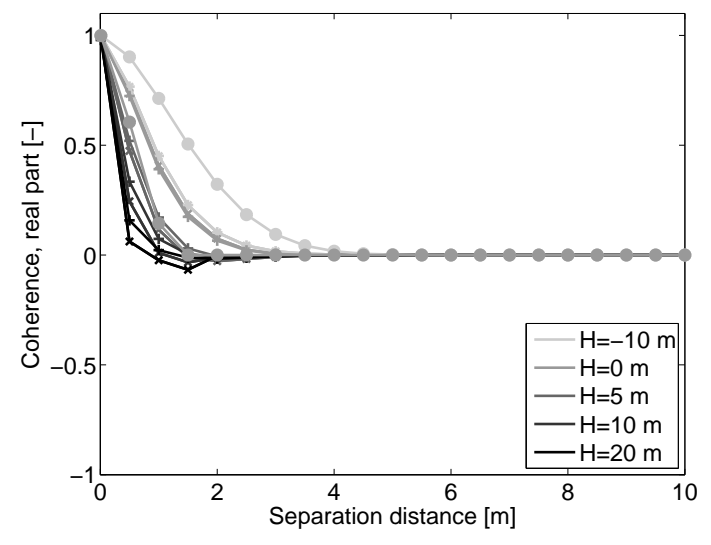

Figure 13: Real part of coherence using the full method (times signs) and the fast method (plus signs) for different screen heights, $H$. The analytical solution is plotted for the two smallest screen heights, $H=-10$ and $H=0 \mathrm{~m}$ (filled circles). $f=2000 \mathrm{~Hz}, L_{0}=100 \mathrm{~m}$ and $C_{v}^{2}=$ $0.1 \mathrm{~m}^{4 / 3} \mathrm{~s}^{-2}, v_{c}=1.2 \mathrm{~m}$ and $v_{\mathrm{F}}=3.9 \mathrm{~m}$. 


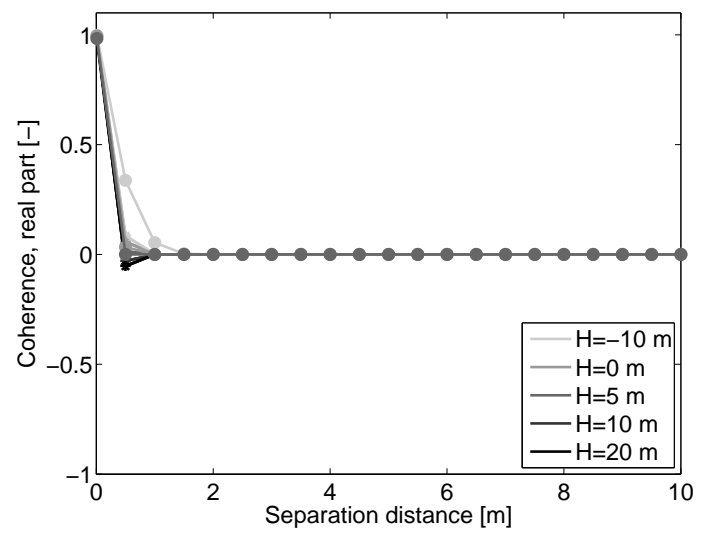

Figure 14: Real part of coherence using the full method (times signs) and the fast method (plus signs) for different screen heights, $H$. The analytical solution is plotted for the three smallest screen heights, $H=-10, H=0$ and $H=5 \mathrm{~m}$ (filled circles). $f=2000 \mathrm{~Hz}, L_{0}=100 \mathrm{~m}$ and $C_{v}^{2}=1 \mathrm{~m}^{4 / 3} \mathrm{~s}^{-2}, v_{c}=0.3 \mathrm{~m}$ and $v_{\mathrm{F}}=3.9 \mathrm{~m}$. 


\section{References}

[1] Forssén, J. An analytical solution for a low barrier in a turbulent atmosphere. AcusticaActa Acustica, Vol. 90, No. 6, 2004, pp. 1142-1150.

[2] Gilbert, K.E., Raspet, R. and Di, X. Calculation of turbulence effects in an upwardrefracting atmosphere. J. Acoust. Soc. Am., Vol. 87, 1990, pp. 2428-2437.

[3] Stinson, M.R., Havelock, D.I. and Daigle, G.A. Comparison of predicted and measured sound pressure levels within a refractive shadow in the presence of turbulence. Proc. Inter-Noise 1995, pp. 327-330.

[4] Daigle, G.A. Diffraction of sound by a noise barrier in the presence of atmospheric turbulence. J. Acoust. Soc. Am., Vol. 71, 1982, pp. 847-854.

[5] Forssén, J. Calculation of sound reduction by a screen in a turbulent atmosphere using the parabolic equation method. Acustica-Acta Acustica, Vol. 84, 1998, pp. 599-606.

[6] Sound shielding in the presence of turbulence (Special issue). Applied Acoustics, Vol. 65, Issue 6, 2004, pp. 559-642.

[7] Daigle, G.A., Piercy, J.E. and Embleton, T.F.W. Effects of atmospheric turbulence on the interference of sound waves near a hard boundary. J. Acoust. Soc. Am., Vol. 64, 1978, pp. 622-630.

[8] Clifford, S.F. and Lataitis, R.J. Turbulence effects on acoustic wave propagation over a smooth surface. J. Acoust. Soc. Am., Vol. 73, 1983, pp. 1545-1550.

[9] Ostashev, V.E., Salomons, E.M., Clifford, S.F., Lataitis, R.J., Wilson, D.K., Blanc-Benon, $\mathrm{Ph}$. and Juvé, D. Sound propagation in a turbulent atmosphere near the ground: A parabolic equation approach. J. Acoust. Soc. Am., Vol. 109, 2001, pp. 1894-1908.

[10] Havelock, D.I., Di, X., Daigle, G.A. and Stinson, M.R. Spatial coherence of a sound field in a refractive shadow: Comparison of simulation and experiment J. Acoust. Soc. Am., Vol. 98, 1995, pp. 2289-2302.

[11] Havelock, D.I., Stinson, M.R. and Daigle, G.A. Measurements of the two-frequency mutual coherence function for sound propagation through a turbulent atmosphere. J. Acoust. Soc. Am., Vol. 104, 1998, pp. 91-99.

[12] Forssén J. and Ögren, M. Barrier noise-reduction in the presence of atmospheric turbulence: Measurements and numerical modelling. Applied Acoustics, Vol. 63, 2002, pp. 173-187. 
[13] Forssén J. Calculation of noise barrier performance in a turbulent atmosphere by using substitute sources above the barrier. Acustica-Acta Acustica, Vol. 86, 2000, pp. 269-275.

[14] Forssén J. Calculation of noise barrier performance in a three-dimensional turbulent atmosphere using the substitute-sources method. Acustica-Acta Acustica, Vol. 88, 2002, pp. 181-189.

[15] L'Espérance, A., Nicolas, J. and Daigle, G. A. Insertion loss of absorbent barriers on ground. J. Acoust. Soc. Am., Vol. 86, 1989, pp. 1060-1064.

[16] Forssén, J. An extended substitute-sources method for a turbulent atmosphere: Calculations for upward refraction. Acustica-Acta Acustica, Vol. 89, 2003, pp. 225-233.

[17] Salomons, E.M. A coherent line source in a turbulent atmosphere. J. Acoust. Soc. Am., Vol. 105, 1999, pp. 652-657.

[18] Abramowitz, M. and Stegun, I.A. Handbook of Mathematical Functions with Formulas, Graphs, and Mathematical Tables. Dover, New York, 1972.

[19] Ostashev V.E. Acoustics in moving inhomogeneous media. E \& FN Spon (an imprint of Thomson Professional), London, 1997. 